\title{
Rastreamento do câncer de mama no Brasil
}

\author{
Breast cancer screening in Brazil \\ Luiz Henrique Gebrim ${ }^{1}$ \\ Luis Gerk de Azevedo Quadros ${ }^{2}$
}

Atualmente o câncer de mama é um problema de saúde pública, não só em países em desenvolvimento, como é o caso do Brasil, mas também em países desenvolvidos, como Estados Unidos e países da Europa Ocidental. Tal situação deve-se à dificuldade de prevenção primária (eliminar fatores de risco ou diagnosticar e tratar lesões precursoras), observando-se como conseqüência aumento significativo na incidência e mortalidade decorrentes desta neoplasia. Segundo a Organização Mundial de Saúde (OMS), observou-se nas décadas de 60 e 70 aumento de dez vezes nas taxas de incidência ajustadas por idade nos Registros de Câncer de Base Populacional de diversos continentes ${ }^{1}$. O aumento na incidência pode ser explicado, em parte, por alterações nos hábitos reprodutivos, como postergação do primeiro parto, e nutricionais (considerando que a obesidade eleva o risco de câncer na pós-menopausa).

Nos Estados Unidos, a American Cancer Society estimou que ocorram em 2006 cerca de 212.930 casos novos e 40.870 mortes por carcinoma de mama. Entretanto, apesar da tendência gradual do aumento na incidência, observa-se nesse país, bem como nos países da União Européia, uma diminuição na mortalidade por esta neoplasia de até $2,3 \%$ ao ano ${ }^{2,3}$.

Apesar da redução da mortalidade por câncer de mama nos Estados Unidos o número de pacientes em estádios avançados não sofreu alteração ${ }^{4}$. A redução provavelmente foi obtida pelo aumento na proporção de mulheres com diagnósticos iniciais (carcinoma "in situ" e estádio clínico I) oriundas de camadas de maior poder aquisitivo, que passaram a realizar a prevenção secundária (diagnóstico na fase pré-clínica por mamografia), aliado à redução de mortalidade obtida pela hormonioterapia adjuvante com tamoxifeno ${ }^{4}$.

No Brasil, o câncer de mama também apresenta alta incidência entre as mulheres. Segundo o Instituto Nacional do Câncer de Ministério da Saúde (INCA), para uma população feminina de cerca de 93 milhões, estima-se que ocorrerão 48.930 casos em 2006, com incidência de 52 casos a cada 100 mil mulheres. Tanto a incidência como a taxa bruta de mortalidade vem apresentando aumento significativo nas últimas décadas ${ }^{5}$, com crescimento de $76 \%$ entre os anos de 1979 e 2004, passando de 5,7 para 10,1 mortes por 100.000 mulheres (Figura 1).

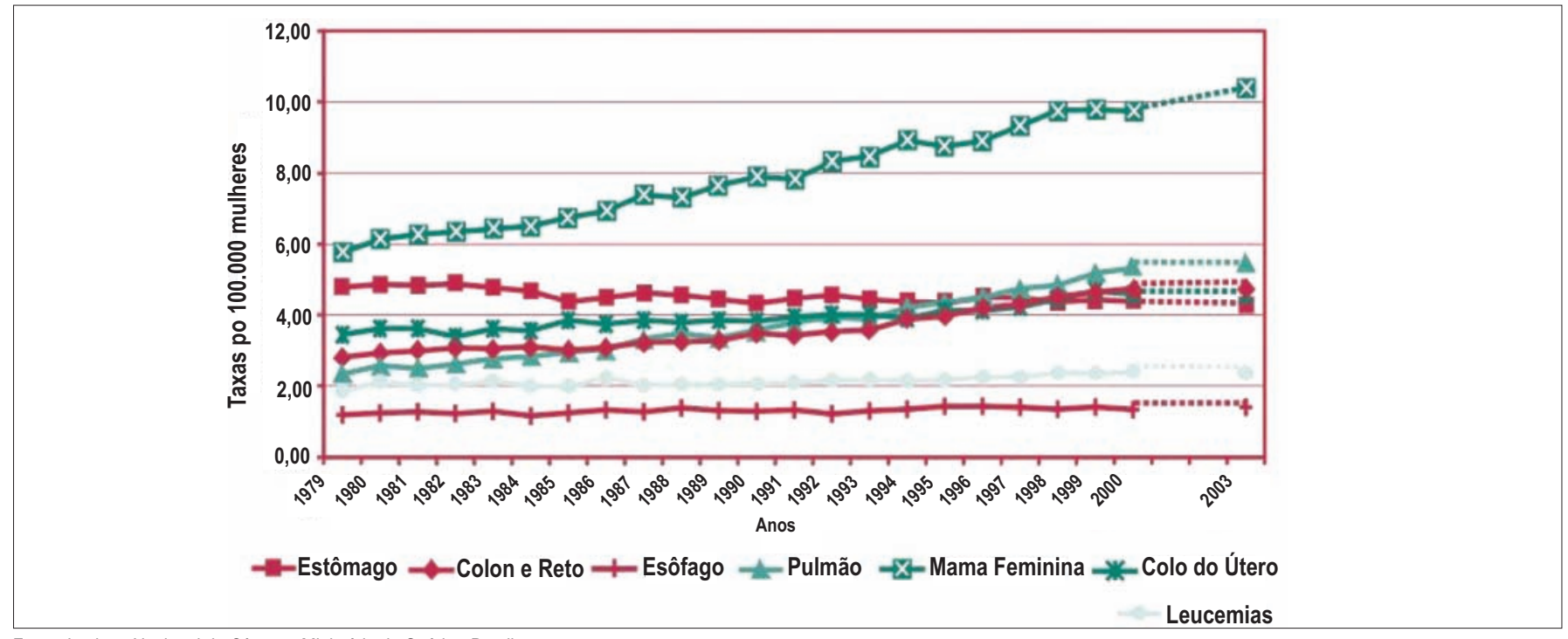

Fonte: Instituto Nacional do Câncer - Ministério da Saúde - Brasil

Estimativa da Incidência e Mortalidade por Câncer no Brasil - 2003

Figura 1 - Mortalidade por Câncer no Brasil (óbitos / 100.000 mulheres-ano).

1 Professor Livre-Docente e Chefe da Disciplina de Mastologia da Universidade Federal de São Paulo - UNIFESP - São Paulo (SP), Brasil; Diretor do Centro de Referência da Saúde da Mulher do Estado de São Paulo - Hospital Pérola Byington - São Paulo (SP), Brasil.

2 Professor do Curso de Pós-Graduação em Ginecologia da Universidade Federal de São Paulo - UNIFESP - São Paulo (SP), Brasil.

Correspondência: Luiz Henrique Gebrim - Alameda Ministro Rocha Azevedo, 1217, $10^{\circ}$ andar - Jardim América - 01410-003 - São Paulo - SP 
Considerando a incidência do carcinoma de mama por regiões e excluindo os tumores de pele não melanoma, a neoplasia de mama é a mais freqüente na região Sudeste, com 73 casos novos por 100.000 mulheres, assim como nas regiões Sul (71/100.000), Centro-Oeste $(38 / 100.000)$ e Nordeste $(27 / 100.000)$. Na região Norte é o segundo tumor mais incidente, com 15 casos novos por 100.000 mulheres.

Entre as principais capitais do país, São Paulo, com 6.170 casos novos, Rio de Janeiro, com 1.230 casos e Porto Alegre, com 1.170 casos novos são os locais onde a incidência do câncer de mama é maior. Tal fato deve-se, além da maior prevalência de fatores de risco nessas cidades, ao atendimento de pacientes advindas de cidades do interior e mesmo de alguns estados do Norte e Nordeste.

As informações do DATASUS mostram que, no ano de 2004, São Paulo foi o estado que apresentou maior mortalidade em números absolutos, com 2.876 óbitos por câncer de mama. Em seqüência, vieram Rio de Janeiro (1.406 óbitos), Rio Grande do Sul (893 óbitos) e Minas Gerais (734 óbitos) (,7. $^{6}$.

O INCA estimou que a cobertura de mamografia, em 15 capitais e no Distrito Federal, variou entre $37 \%$ e $76 \%$. Entretanto, o percentual de realização de mamografia pelo Sistema Único de Saúde (SUS) variou entre $17 \%$ e $54 \%$ do total, o que em parte explica o diagnóstico tardio e as altas taxas de mortalidade ${ }^{8,9}$.

Com base nestes dados foi elaborado em 2004 um documento de consenso para a detecção precoce do câncer de mama no Brasil. As decisões foram tomadas de acordo com as evidências científicas obtidas de programas adotados pelos países que desenvolvem políticas públicas, adaptadas à realidade da infra-estrutura brasileira. A diretriz preconiza a realização do exame clínico das mamas anualmente a partir dos 40 anos e rastreamento mamográfico bienal dos 50 aos 69 anos. Nas mulheres de alto risco, preconiza-se exame clínico e mamografia anuais a partir dos 35 anos.

Apesar de o rastreamento mamográfico permitir redução de mortalidade de até $18 \%$ nas mulheres com idade entre 40 e 50 anos, a redução supera $30 \%$ após os 50 anos e a relação custo beneficio é mais favorável nesta população mais idosa ${ }^{10}$. O rastreamento com mamografia anual a partir dos 50 anos, adotado há vários anos pelo Canadá e países Europeus, visa priorizar a população de maior incidência da neoplasia. Se considerarmos que $62 \%$ das mulheres da região Sudeste com idade superior a 60 anos jamais foram submetidas a uma mamografia, há de se priorizá-las numa primeira etapa.

A ampla divulgação pelos meios de comunicação da importância do diagnóstico precoce modificou o conceito de que a falta de conscientização e o medo de câncer eram os principais responsáveis pelo grande número mulheres com tumores avançados no Brasil. Tais medidas vêm acarretando cancerofobia em jovens, pelo desconhecimento de que a neoplasia é rara antes dos 35 anos e pelo constante exposição na mídia de mulheres jovens com a doença. Tal distorção acarreta um acúmulo de pacientes sem doença nos Centros de Referência, muitas vezes com mais de uma mamografia realizadas precocemente sem necessidade, com achados negativos ou benignos, em detrimento de mulheres mais idosas, muitas com doença clínica, que aguardam meses por atendimento.

Temos constatado que o grande desafio atual decorre da falta de acesso aos poucos Centros especializados, que por sua vez nem sempre estão capacitados para diagnóstico e tratamento rápido. Além de escassos e mal distribuídos, os Centros de Referência atuam com recursos humanos e infraestrutura subutilizados. A falta de um programa nacional regionalizado e hierarquizado para detecção precoce dificulta o gerenciamento das ações e a capacitação médica, sendo freqüente a migração de pacientes provenientes de áreas com atendimento deficiente (outros estados e interior), sobrecarregando e onerando os mais centros ágeis e de fácil acesso, como ocorre na capital de São Paulo. A maior concentração de recursos materiais e humanos no município e a grande população de migrantes que lá residem, contribuem para que a metrópole tenha maior número de óbitos decorrentes de câncer de mama.

Apesar dos esforços para aumentar o número de mamógrafos para se detectar tumores não palpáveis, o tempo médio para diagnóstico e início do tratamento das pacientes com tumores palpáveis supera 120 dias. $O$ atendimento da rede primária é deficiente, faltam diretrizes, treinamento e acesso rápido para o primeiro atendimento. O médico, por sua vez, solicita exames muitas vezes desnecessários, que oneram e sobrecarregam os Centros de Referência.

Esta distorção dificulta o acesso das pacientes, retarda a elucidação das queixas, eleva o nível de ansiedade das mulheres, principalmente das que não têm câncer, e, obviamente, retarda o diagnóstico, com piora no prognóstico. A terapêutica dos casos avançados é mais onerosa e as chances de cura são inferiores a $30 \%$. 
Tais evidências mostram claramente que antes de se desencadear ações no sentido de detectar precocemente o câncer de mama, temos que priorizar a paciente com tumor palpável, mapeando os Centros de Referência que efetivamente são atuantes para facilitar o acesso à rede pública, que deve estar capacitada e hierarquizada para o diagnóstico resolutivo.

Uma tentativa de reverter essa realidade em curto prazo seria aperfeiçoar os recursos já existentes, ou seja, disponibilizando as equipes de atendimento de forma integrada e multidisciplinar, direcionandoos para o atendimento resolutivo em consulta única. Valendo-se de critérios técnicos, há necessidade da distribuição de responsabilidades entre as unidades de atendimento primário e secundário, em total sincronismo com os Centros de Referência (terciários) para a efetiva triagem das pacientes sintomáticas.

A Secretaria Estadual da Saúde de São Paulo vem desenvolvendo, em cooperação com o Centro de Referência da Saúde da Mulher, a Disciplina de Mastologia da UNIFESP e a Fundação Oncocentro, um plano estratégico para redução de mortalidade em câncer de mama. O programa visa duas prioridades: dar resolutividade diagnóstica e tratamento ágil às pacientes com alterações clínicas e secundariamente a realização do rastreamento mamográfico.

Para a primeira, implantou-se no Hospital Pérola Byington um Centro de Alta Resolutividade em Mastologia (CARE) com atendimento em local dotado de mamógrafo, ultra-sonografia e microscópio para exame citológico com coloração imediata, disponibilizando-se agulhas para biópsias percutâneas e sala para cirurgia ambulatorial. O atendimento em consulta única é coordenado por mastologistas treinados em atendimento resolutivo, em conjunto com médicos ginecologistas e residentes. A equipe atende toda a demanda diária de consultas novas (cerca de 80 ao dia e 1.600 por mês). O modelo de atendimento tem finalidade não só assistencial, mas também de ensino, capacitando futuros especialistas para atuar desta forma prioritariamente na rede pública.

As pacientes são submetidas a um breve questionário para identificar as unidades básicas que as encaminharam para a contra-referência caso necessário. Após o exame fisico para confirmação de alterações clínicas, procede-se, a critério médico, à propedêutica imagenológica, punção biópsia com coloração e leitura imediata da lâmina. Nos casos duvidosos, realiza-se a biópsia por agulha grossa ou incisional. As pacientes com câncer têm seus exames pré-operatórios ou pré-quimioterapia solicitados e a cirurgia ou início da quimioterapia são agendados para um período máximo de 15 dias.

O planejamento desse tipo de ação acoplada ao Centro de Alta Resolutividade (CARE) supera o mais importante obstáculo para se obter redução na mortalidade, pois aumenta a resolutividade das complexas e dispendiosas etapas do diagnóstico, evitando exames desnecessários e propiciando terapêutica imediata e individualizada. Elimina também a ansiedade da espera de resultados, humanizando o atendimento tanto na rede pública como privada.

Esse trabalho visa identificar e capacitar as equipes da capital e interior para descentralizar e viabilizar os programas de rastreamento mamográfico das pacientes assintomáticas com idade superior a 50 anos. Ao analisar as informações referentes a mais de 5.000 mulheres atendidas neste Centro em 2005, observamos que o atendimento resolutivo permitiu concluir o diagnóstico em lesões clínicas em $75,4 \%$ das pacientes. Cerca de $34 \%$ das pacientes encaminhadas não tinham nenhuma doença mamária e, destas, $62 \%$ tinham realizado exames por imagem solicitados pelo ginecologista.

Os dados revelaram um grande contingente de casos com encaminhamentos e exames desnecessários, evidenciando a necessidade da realização de cursos de capacitação para os médicos que atuam na rede primária. O atendimento resolutivo, por outro lado, abreviou o período de ansiedade das pacientes cancerofóbicas. As demais causas de encaminhamento foram alterações não palpáveis $(25 \%)$ fibroadenomas (18\%) e cistos $(15 \%)$, correspondendo os carcinomas a apenas $7 \%$ do total de pacientes atendidas.

Tendo em vista o grande número de alterações mamográficas e doenças benignas, há necessidade de qualificar os hospitais ou unidades secundárias de contra-referência para triagem e resolução desses casos, evitando o deslocamento da paciente ou a realização de exames subsidiários desnecessários. Ressalte-se que cerca de 30\% das mulheres atendidas no Hospital Pérola Byington são do interior ou de outros estados da União.

Outro aspecto importante observado foi o predomínio de mulheres jovens, com idade próxima dos 38 anos, mostrando maior conscientização nesse grupo etário. Temos que corrigir esta distorção, incentivando as jovens a trazer as mais idosas para o rastreamento mamográfico, pois as últimas estão menos conscientizadas, sendo muitas vezes dependentes de algum familiar para acompanhamento à consulta. A proporção de mulheres com tumores nos estádios clínicos I e II aumentou de 41 para 65\%. Ao contrário, a proporção de casos avançados (estádios III e IV) foi reduzida de 55\% para 34\%. 
Este resultado mostra de forma clara que a falta de acesso e de resolutividade é uma das mais importantes causas de progressão da doença, pois, em 3 ou 6 meses, grande parte das neoplasias das pacientes pertencentes ao estádio II evoluiria para III ou IV. Vencer essa etapa é primordial antes de se iniciar o rastreamento mamográfico.

No tocante ao rastreamento, a Secretaria Estadual da Saúde realizou dois grandes mutirões de mamografia no Estado de São Paulo. Foram rastreadas mais de 150.000 mulheres em 2005 e 80.000 em maio de 2006. Trata-se da primeira experiência em rastreamento populacional no Brasil, para o qual foram utilizados mais de 360 serviços públicos e terceirizados dotados de mastologistas e mamógrafos.

Resultados parciais de 51.000 exames mostraram proporção de alterações (Categorias BI-RADS ${ }^{\circledR} 4$ e 5) semelhante à dos programas europeus. O resultado de 14,5\% de categoria BI-RADS ${ }^{\circledR}$ zero (necessidade de exame imagenológico complementar), superou os da literatura (Tabela 1). Tal fato pode ser atribuído à falta de padronização de leitura pelos radiologistas, mostrando mais um a vez a necessidade de capacitar as equipes envolvidas nas diferentes etapas do rastreamento e no controle de qualidade dos serviços credenciados. O número de casos de carcinoma esperado para uma população de mulheres com idade superior aos 50 anos oscila entre 3 e 6 para cada 1.000 exames. Os resultados mostraram uma proporção de 3 por 1.000, provavelmente pelo grande contingente de mulheres com idade entre 40 e 50 anos. Em 2006, enfatizou-se a prioridade para mulheres que nunca haviam feito o exame, em especial aquelas com mais de 50 anos.

No momento, o Brasil não pode implantar o rastreamento mamográfico em todo o território pela falta de recursos econômicos para o exame e, principalmente, de infra-estrutura para a continuidade investigacional das lesões não palpáveis. Grande parte dos estados da federação, inclusive da região sudeste, só disponibiliza o exame para diagnóstico.

Em resumo, o câncer de mama é considerado uma doença de bom prognóstico desde que diagnosticado e tratado precocemente. Apesar de estudos observacionais indicarem que a prevenção da obesidade diminua o risco da doença na pós-menopausa, vem se dando ênfase à prevenção secundária com o intuito de melhorar a sobrevida das pacientes ${ }^{12}$. Enquanto em países desenvolvidos o auto-exame das mamas já não exerce importância na detecção precoce do câncer mamário por não ter impacto sobre a mortalidade, nas normas para controle do câncer de mama no Brasil o INCA preconiza tal medida apenas como recurso para conscientização da mulher. A experiência em atendimento resolutivo vem sendo adotada em cinco pólos estaduais, pois propicia imediata redução do número de casos avançados e humaniza o atendimento na rede pública, utilizando recursos humanos e materiais existentes e dispersos. Contribui a médio e longo prazo para o efeito multiplicador de ações integradas e resolutivas, no ensino e capacitação de médicos especialistas, voltados para a realidade de cada estado brasileiro.

Nossa prioridade deve ser investir em programas de capacitação médica, hierarquizar e garantir acesso rápido aos centros secundários e terciários para atendimento resolutivo, propiciando condições de fluxo efetivo para o rastreamento da doença, objetivando em médio prazo uma efetiva redução da mortalidade do câncer. Um programa de rastreamento no Brasil deverá ser implantado apenas em regiões dotadas de infra-estrutura ágil e capacitada a investigar lesões não palpáveis, assegurando recursos econômicos adicionais para a terapêutica complementar do carcinoma (radio, quimio e hormonioterapia).

Tabela 1 - Resultados parciais do mutirão de mamografia realizado no Estado de São Paulo: distribuição de casos de acordo com a classificação pelo sistema BI-RADS ${ }^{\circledR}$ (Fundação Oncocentro, 2005) ${ }^{11}$.

\begin{tabular}{lcc}
\hline $\begin{array}{l}\text { Classificação } \\
\text { Bl-RADS }^{\circledR}\end{array}$ & (N) & (\%) \\
\hline 0 & 7.247 & 14,4 \\
1 & 20.296 & 40,5 \\
2 & 19.814 & 39,5 \\
3 & 2.433 & 4,9 \\
4 & 312 & 0,6 \\
5 & 44 & 0,1 \\
Total & $\mathbf{5 0 . 1 4 6}$ & \\
\hline
\end{tabular}




\section{Referências}

1. World Health Organization (WHO) [homepage on the Internet]. World health statistics annual: 1997-1999. WHO; 2001 [cited 2006 Jun 26]. Available from: http://www.who.int/en/

2. Althuis MD, Dozier JM, Anderson WF, Devesa SS, Brinton LA. Global trend in breast cancer incidence and mortality 1973-1997. Int J Epidemiol. 2005;34(2): 405-12.

3. Boyle P , Ferlay J. Cancer incidence and mortality in Europe, 2004. Ann Oncol. 2005; 16(4): 481-8.

4. Schootman M, Jeffe D, Reschke A, Aft R. The full potential of breast cancer screening use to reduce mortality has not yet been realized in the United States. Breast Cancer Res Treat. 2004; 85(3): 219-22.

5. Instituto Nacional do Câncer (INCA). Estimativa da incidência e mortalidade por câncer no Brasil [monografia na Internet]. Brasília (DF): Ministério da Saúde; 2004. [citado 2006 Jun 26]. Disponível em: http://www. inca.gov.br/estimativa/2006/

6. Instituto Brasileiro de Geografia e Estatística (IBGE) [sítio na Internet]. Diretoria e Pesquisa. Departamento de População e Indicadores Sociais. População residente - Censo 2000: Brasil, unidades da federação e municípios, 2000 [citado 2006 Jun 26] Disponivel em: http:/ /www.ibge.gov.br/

7. Departamento de Informática do SUS. Informações de saúde. Estatísticas vitais - mortalidade e nascidos vivos. Disponivel em: http://www.datasus.gov.br

8. Paulinelli RR, Freitas Júnior R, Curado MP, Souza AA. A situação do câncer de mama em Goiás, no Brasil e no mundo: tendências atuais para a incidência e a mortalidade. Rev Bras Saúde Matern Infant. 2003; 3(1):17-24.

9. Thuler LCS, Mendonça GA. Estadiamento inicial dos casos de câncer de mama e colo do útero em mulheres brasileiras. Rev Bras Ginecol Obstet. 2005;27(11): 656-60.

10.Smith RA, D'orsi CJ. Screening for breast cancer. In: Harris JR, Lipmann ME, Morrow M, Osborne CK, editors. Diseases of the breast. Philadelphia: Lippincott; 2005. p.103-30.

11.Fundação Oncocentro de São Paulo [homepage da Internet]. Rastreamento mamográfico no Estado de São Paulo - resultados preliminares de 2005. São Paulo; 2005 [citado 2005 Ago 29]. Disponível em: http:/ / www. fosp.saude.sp.gov.br

12. Souza Pinho VF, Coutinho ESF. Fatores de risco para câncer de mama: uma revisão sistemática de estudos com amostras de mulheres da população geral do Brasil. Cad Saúde Pública. 2005;21(2):351-60. 